\title{
Civil Society's Contribution to Rolling out of Option B+ in Malawi - A Case of Umunthu Foundation
}

\author{
David Odali ${ }^{1, *}$, Yohane Gadama ${ }^{2}$ \\ ${ }^{1}$ Umunthu Foundation, Malawi \\ ${ }^{2}$ Queen Elisabeth Central Hospital (QECH), Malawi
}

Copyright $\mathrm{O} 2017$ by authors, all rights reserved. Authors agree that this article remains permanently open access under the terms of the Creative Commons Attribution License 4.0 International License

\begin{abstract}
Option B+ was developed in Malawi in 2010 and adopted in 2011 in response to the impracticality of implementing the World Health Organization (WHO) 2010 guidelines on prevention of mother-to-child transmission (PMTCT) of HIV. The approach was deemed very ambitious in a Malawian setting given the ongoing service delivery challenges. Major challenges included shortage of human resources (qualified health staff), lack of proper infrastructure, stigma associated with taking antiretroviral drugs, shortages of HIV test kits and related accessories. Amidst these challenges, civil society organizations like Umunthu Foundation (UMUFO) partnered with government to better serve mothers living with HIV. UMUFO actively worked with communities to enhance awareness of Option $\mathrm{B}+$ and aide in retaining women in care once initiated on ART. UMUFO has particularly been working with two public health facilities (Limbe and Bangwe Health Centers) in providing HIV testing services, infrastructural needs and hiring health workers. With these services, UMUFO has managed to maintain a significant increase of women tested and started on Option B+ by almost 200\% (from 408 in $2014 / 2015$ to 1219 women in 2016/2017) in 3 years. The impact UMUFO has had on rolling out of Option B+ clearly demonstrates the critical role civil society organizations can have in meeting national and global public health targets.
\end{abstract}

Keywords Option B+, PMTCT, Mentor Mothers, Antenatal, Civil-society Organizations, Umunthu Foundation, Malawi

\section{Background}

In 2011, the United Nations Political Declaration on HIV and AIDS established commitments and targets, including eliminating new HIV infections among children and substantially reducing AIDS-related maternal deaths by 2015 [1]. Despite these commitments, in 2012, new pediatric HIV infections were still high $(260,000$ new infections) and $40 \%$ of pregnant women in 20 countries with a high burden of HIV infection were not receiving antiretroviral (ARV) drugs to prevent the vertical transmission of HIV [2].

In 2010, WHO recommended that HIV+ pregnant women who are eligible for antiretroviral therapy (ART) receive lifelong triple ARV treatment. The eligibility for ART was based on a CD4+ T lymphocyte (CD4+ cell) count of $\leq 350$ cells $/ \mu 1$, or on the presence of WHO stage 3 or 4 diseases. The guidelines also recommended that HIV positive pregnant women not eligible for lifelong ART be placed on one of two PMTCT protocols - Option A or Option B beginning as early as the 14 th week of gestation. This was aimed at reducing the risk of vertical transmission of HIV during pregnancy, delivery and the postpartum period including breast-feeding [3].

The Option A protocol comprised giving zidovudine (AZT) to the mother prophylactically during pregnancy, single-dose nevirapine (NVP) to both mother and neonate at delivery, maternal AZT and lamivudine (3TC) to the mother during the first week postpartum, and NVP daily to the infant throughout breastfeeding. The Option B protocol comprised a triple ARV regimen, typically consisting of the recommended first-line ART for the mother during pregnancy and throughout breastfeeding, plus 6 weeks of daily NVP for the infant, regardless of infant feeding method [3].

As much as the use of ART proved to be efficacious as recommended by $\mathrm{WHO}$, countries like Malawi struggled to adopt and fully implement either Option A or Option B. The limitation hinged on difficulties Malawi had to provide reliable CD4 count testing for HIV positive women. Nearly $70 \%$ of pregnant women were attending antenatal care services at smaller health facilities where reliable CD4 counting was not available and would not be available for years to come [4]. The WHO guidelines were thus not viable in Malawi as they were critically dependent on the availability of CD4 count testing.

To better serve its population, the Ministry of Health $(\mathrm{MoH})$ through the department of HIV and AIDS implemented an innovative public health approach for 
pregnant women with HIV. The approach, dubbed 'Option B+', involves starting all HIV positive pregnant or breast-feeding women on lifelong ART regardless of their clinical stage or CD4 count. Since the Option B+ approach did not require $\mathrm{CD} 4$ count, it appeared to be a game changer as it led to a dramatic increase in the number of HIV positive pregnant women started on ART (763\%), with retention rates at twelve months (78\%). [5]. A 7-fold increase in ART initiation in pregnant and lactating mothers was also reported [6]. These results were observed barely after eighteen months of implementation. Since then, Option B + grew its popularity and proved to be replicable, scalable, cost-effective and efficacious for other countries. As such, WHO, recommended Option B +in its 2015 guidelines for PMTCT

\section{Challenges of Implementing Option B+}

Implementation of Option B+ in Malawi brought its own challenges. As could be expected, the increased numbers of women started on ART meant an increased workload for the already fatigued and under-staffed health facilities in Malawi. The $\mathrm{MoH}$ tried to circumvent the problem by increasing the number of sites offering ART from 350 to over 650 . However, congestion for Option B+ still became a common sight at most of the accredited public health facilities. The increasing number of women seeking HIV testing and subsequent start-up of ART (if found positive) created another strain on the government in terms of providing HIV test kits and accessories, and trained HIV testing counsellors.

Another challenge was public acceptance and community support for ART for life. Women were afraid of disclosing their HIV status because the stigma and discrimination that came with the disclosure $t$ had a negative impact on adherence and retention in care. A national-wide study done in Malawi, looking at facility-level data, found that nearly $24 \%$ (95\% CI 22.6-25.3\%) of women started on ART while pregnant are lost to follow-up in the first 6 months [7]. These findings were consistent with findings of other observational studies done Sub-Saharan Africa in showing the gaps in retaining women on care once initiated on Option $\mathrm{B}+[8,9]$.

\section{Contribution from Civil Society Organizations (CSOs)}

To ensure maximal benefit of the Option $\mathrm{B}+$ strategy, a number of civil society organizations (CSOs) joined hands with the $\mathrm{MoH}$ to provide services to citizens. One of such organizations was UMUFO, a community-based organization working in the townships of Bangwe and Limbe in Blantyre, Malawi's commercial city. In partnership with a UK-based HIV and AIDS international charitable organization called AVERT, UMUFO has been providing
HIV services in these areas since 2010. UMUFO uses a rights-based approach (RBA) to promote HIV prevention, treatment, care and support [10].

UMUFO has contributed to the rolling out of Option $\mathrm{B}+$ in Malawi through the following ways:

\section{a) Community Awareness}

To be successful, Option B+ needed to be embraced by both women and men, and community leaders. UMUFO run awareness campaigns on Option $\mathrm{B}+$ which is disseminated through the radio health programs, and radio interviews. Special awareness campaigns are carried during special events like during the commemoration of the World AIDS Day (WAD), International AIDS Candlelight Memorial (IACM) and 16 Days of Activism against Gender Based Violence. The awareness campaign messages include information on mode of HIV transmission from HIV infected mother to her baby and emphasis is on how HIV positive pregnant women can prevent the transmission through Option $\mathrm{B}+$. Awareness campaign messages are disseminated through targeted Information, Education and Communication (IEC) materials such as posters and brochures which are distributed for free to the pregnant women and their spouses. In addition, dramas and poems are performed live during community gatherings whereas video-recorded materials are played before clients in their waiting shelters at all HIV testing service clinics. Further, before receiving antenatal services, all pregnant women in attendance attend motivational talk sessions which highlight the importance of Option B+ to both the mother and the baby. Special awareness programs with information on Option B+ are aired through community radio health programs. During the annual commemoration of World AIDS Day (WAD) and International AIDS Candlelight Memorial, messages on Option $\mathrm{B}+$ are disseminated across the UMUFO supported areas of Bangwe and Limbe townships through focused group discussions, interface meetings among community health champions (community voluntary care givers), client experts who track down the lost-to-follow clients and health providers from the public health facilities. Such forums prove effective on addressing challenges which could affect the success of Option B+. In collaboration with other public and private stakeholders, UMUFO also provides support in producing banners which carry clear information on live testimony sessions on Option $\mathrm{B}+$, providing a platform to volunteers who explain how they benefited from Option B+.

\section{b) Enhancing Social Support Systems}

Studies done in Malawi and across Africa on why many women are lost to follow-up have demonstrated that poor social support from the husband and family relatives strongly predicts poor adherence to ARTs. A study done at Bwaila Hospital in Malawi found that for women who were in relationships, an important aspect of their support system was having a supportive husband or partner. Partners aided women's retention and adherence by motivating them to start 
swallowing the prescribed ARVs, reminding them to take their drugs daily, allaying treatment-related fears, and assisting them financially to remain in care (including provision of transport money) [11].

Realizing that the health workers are already overstretched with hospital work, UMUFO took it upon itself by going into communities visiting pregnant and breastfeeding women in their homes to strengthen the social support. Among others, UMUFO home visits aim at engaging the male partners in the on-going care and hospital follow-up visits. This has also been demonstrated in PMTCT programs where male partner involvement has been shown to statistically increase women's likelihood to accept HIV testing, initiate treatment, remain in care, and decrease the risk of infant death due to vertical transmission $[12,13,14]$.

Through home visits, it was evident that getting initiated on Option $\mathrm{B}+$ is a psychologically and emotionally complex process for women. This concurs with what was found by [11] and this complex stage has been described as a "triple burden": transitioning into pregnancy, accepting their HIV diagnosis, and acknowledging the immediate need to begin lifelong ART, often while asymptomatic [15]. Such a triple burden could only be lessened through continual support from family. Therefore, thorough counselling and the home visits were just on point at meeting this need.

\section{c) Mentor Mothers}

Through the community engagement and home visits, true stories from mothers who gave birth to HIV negative babies (followed up to the point of being declared HIV negative), are used to emphasize the benefit of Option $\mathrm{B}+$ and the importance of accessing its services during and after pregnancy. Some willing women are even involved in motivating and mentoring other young mothers who in most cases struggle to start ART and adhere to treatment. This usage of mentor mothers has proven to provide the needed support and motivation [16].

Despite this approach proving to be very effective, UMUFO has not fully utilized this due to limitation of its capacity to get more mentor mothers and give them a minimum incentive.

\section{d) Input in Formulation of by-laws}

Through its involvement with local leaders, Umunthu Foundation worked with community leaders and local authorities at the Blantyre City and District Councils to formulate by-laws to sanction women and their husbands opting to deliver their babies at traditional birth attendants (TBAs) rather than at health facilities. Through such active community sensitization and awareness, TBA services became almost completely unreported as women understood the importance of antenatal and hospital delivery as an opportune window to prevent HIV transmission to the child and to reduce maternal mortality rates. The women who are now more knowledgeable of the need and importance of giving birth at a health facility appreciate the difference if compared to the time they were giving birth in their homes or at the traditional birth attendants.

\section{e) Human Resources}

In 2012 and 2013, UMUFO with support from AVERT extended its HIV and AIDS project activities to Bangwe and Limbe Health Centres, respectively, in addition to their own clinic, where HIV testing services had been facing challenges due to understaffing and increased demand for Option $\mathrm{B}+$. The government employed nurses and midwives had already been over-burdened with work focusing on other ailments and could hardly find time to do the HIV testing and counselling. The Health Surveillance Assistants (HSAs) who were trained in HIV counseling and testing, and deployed in public health facilities, could also not provide adequate services as they were absorbed into the other support services to the nurses and midwives. Consequently, this acute shortage of special health personnel who could provide HIV testing and counseling services affected the pregnant women who could spend more time at a health facility before accessing the services, in which case the services could most of the times not be accessed as the women could not wait the whole day. The next day, they could simply not come again for fear of spending the whole day without getting the services or getting it late in the day.

Through the project, additional HIV counseling and testing staff members were employed and deployed in all the project supported health facilities. The project staff could provide HIV testing and counselling services to patients who came for through the out-patient department (OPD) and PMTCT Option B+. The project staff could also take blood samples for Dried Blood Spot-Polymerase Chain Reaction and viral load. Project staff tested pregnant women and conducted early infant diagnosis tests for laboratory testing at the public and private laboratories. The availability and accessibility of HIV testing services motivated more pregnant women to come forward for anti-natal services realizing that they would get the services on the same day. More pregnant women could test for HIV under Option B+ in all the UMUFO supported health facilities.

The project staff members that were trained under the government-regulated training courses on HIV counseling and testing worked under regular supervision by the HIV and AIDS Unit of the Ministry of Health to ensure quality control for quality service delivery. During supervisions, the services provided by the health facilities were proven to be of the required standard. On every quarterly supervision, the health facilities were awarded with certificates of excellence in HIV testing services. Limbe Health Centre, one of the three health facilities where the project staff work was awarded with a certificate of excellence in HIV testing services including the ART service delivery.

\section{f) Infrastructural Contribution}

The increased number of pregnant women initiating ART and other ART patients created space shortage and the lack 
of a waiting shelter was hampering HIV and AIDS service delivery. The shortage of space was even made worse with the introduction of the 'Test and Treat' strategy which entails prompt initiation of ART in everyone tested HIV positive regardless of CD4 count or clinical staging. Patients could arrive at the health facilities as early as four o'clock in the morning in anticipation of getting the services earlier but could spend the whole day being before being attended to as they were queuing for one single room to get HIV testing services and another single room to access ART.

With increased demand for HIV testing services, there were no waiting shelters for patients before accessing the services; they could remain standing in queues even on the rainy, sunny and cold days. One day, during our routine supervision at Limbe Health Centre, we witnessed one patient collapsing and falling to the ground. On enquiry, we were told that the patient had arrived at the facility early in the morning but and waited while standing since then until afternoon without being attended to due to inadequate space and because ART drugs were dispensed from one tiny room. This prompted our efforts to mobilize resources from our UK-based partner, the AVERT to construct a new ART clinic and waiting shelter to decongest the queues.

In 2016, in response to the need for conducive space for HIV testing and treatment services at Limbe Health Centre,
UMUFO constructed a new ART Clinic and ART waiting shelter at the Centre with support from AVERT. The new facility provides adequate space for all ART patients including to-be-seen pregnant women and a comfortable waiting area providing shade from the sun and shelter from the rain.

With a new ART Clinic which increased space for HIV testing and treatment from two to seven rooms, including a separate registration room and waiting shelter, the number of people tested for HIV doubled from 16,527 in 2014/2015 to 33,231 in 2015/2016 which further increased to 36,898 at a close of 2016/2017 (Fig. 1). The increase in numbers is also true for pregnant women tested for HIV through regular antenatal clinics in the health facilities where UMUFO is working. As can be seen from Fig 2, there is a progressive increase in the number of pregnant women tested HIV positive and promptly initiated on Option $\mathrm{B}+$; from 408 women in 2014/2015, the number has almost tripled to 1219 in $2016 / 2017$. This is a significant contribution to the fight against HIV as it contributes to the elimination of mother to child transmission of the virus. Overall, with the aforementioned interventions carried by UMUFO the numbers of both women tested and started on Option $\mathrm{B}+$ are on a continuous increasing trend.

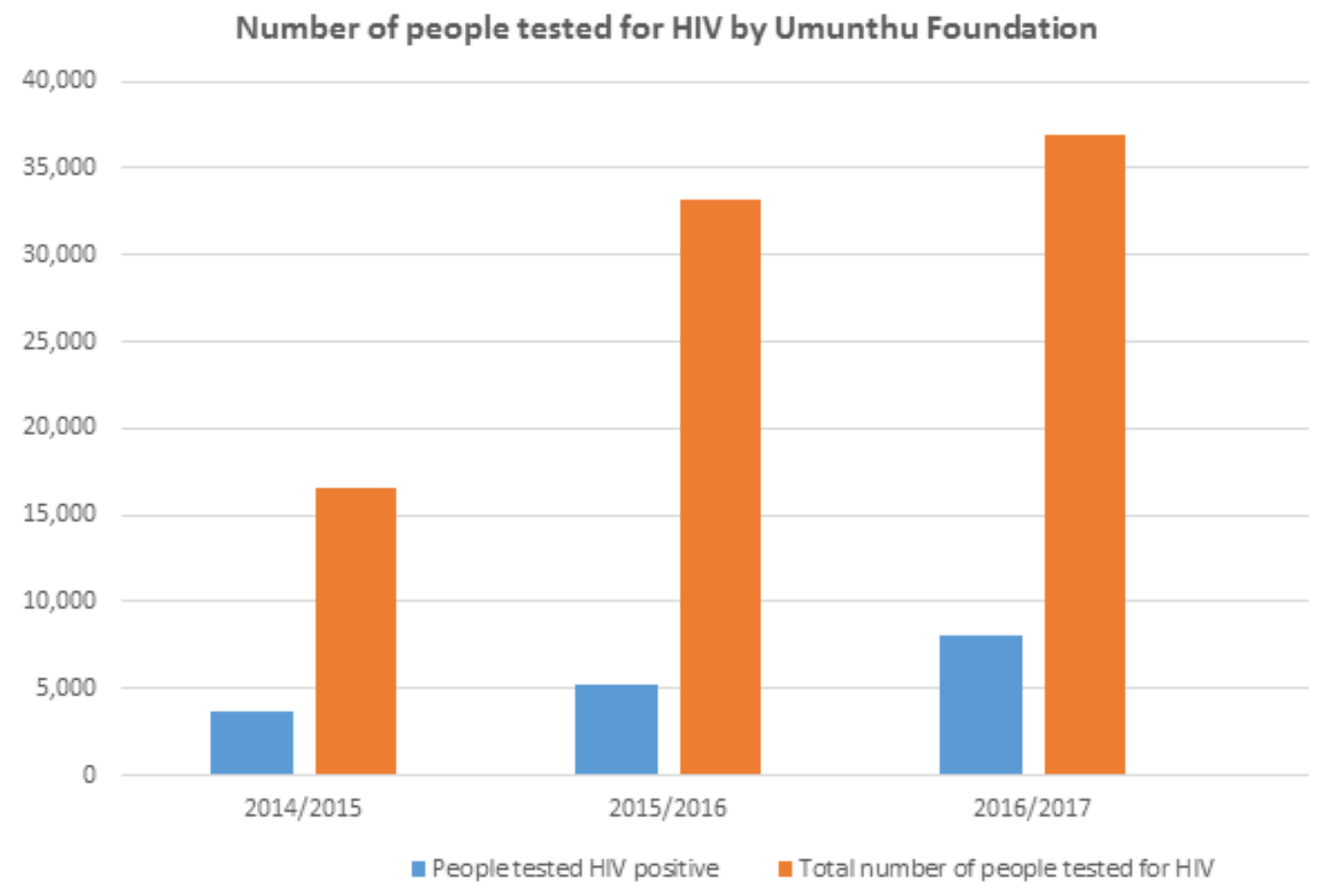

Figure 1. Number of people tested for HIV by Umunthu Foundation over the past 3 years 


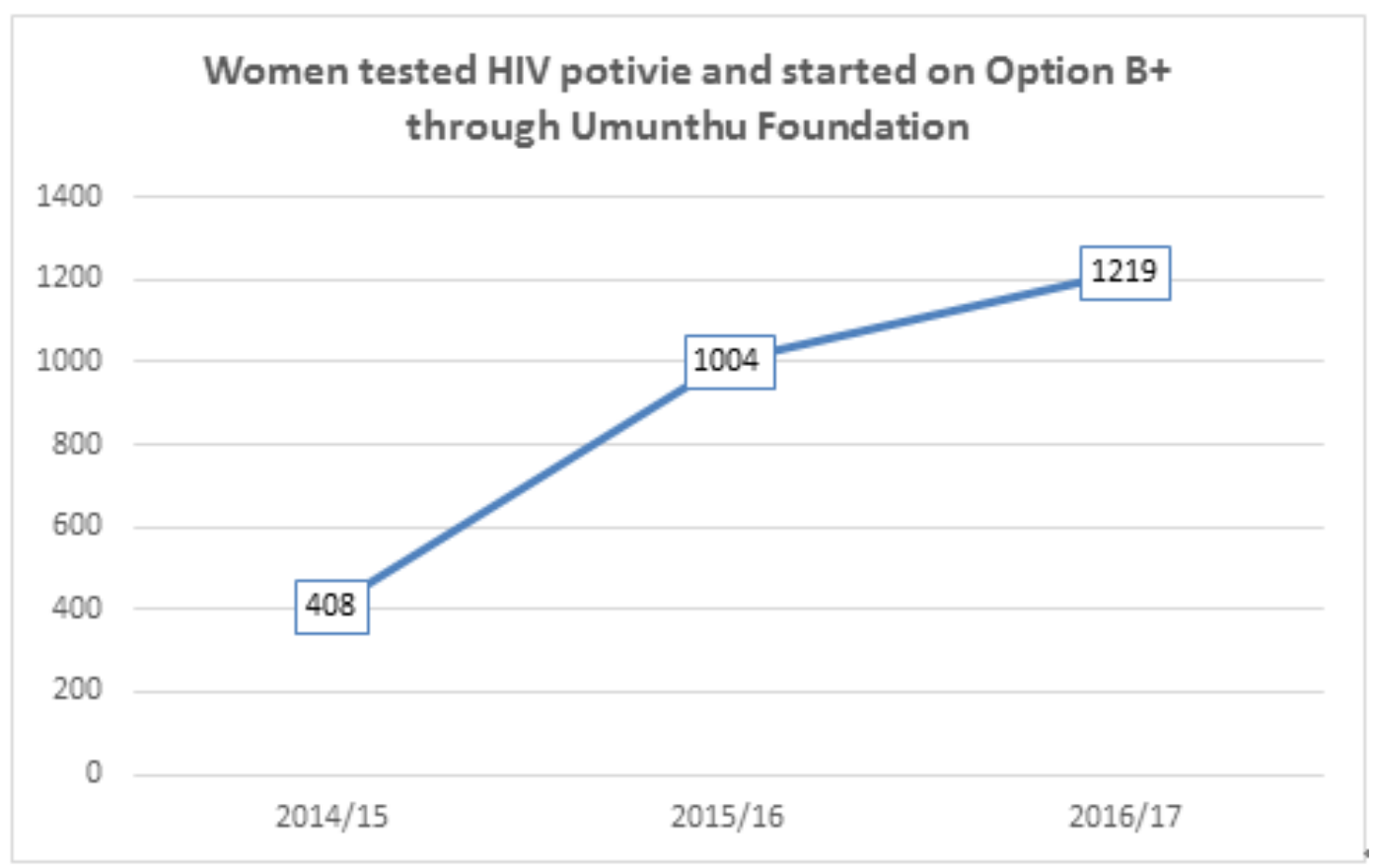

Figure 2. Women tested HIV positive and started in Option B+ by Umunthu Foundation over the past three years,

\section{Conclusions and Way Forward}

Option $\mathrm{B}+$ which was championed by the Ministry of Health in Malawi through its HIV and AIDS department is a role model approach which creates a huge impact in HIV prevention of mother-to-child transmission. Umunthu Foundation, a civil society organization, has made immense contributions towards the success of the program through provision of additional human resource, constructing a conducive infrastructure to deliver services and creating public awareness which together made a positive impact on the successful implementation of the Option $\mathrm{B}+$ in Malawi. As evidence shows, Option B + is a reality that the 'Test $n$ Treat' approach and the ambitious UNAIDS 90-90-90 target can be met provided all players play their roles. Other players, besides government should be involved and be given room to contribute towards achieving an HIV-free generation. In response to HIV, civil society action has been central to many of the milestones achieved as outlined in the Geneva: UNAIDS 2015. Community-based organizations have proven critical in overcoming many of the major challenges in the AIDS response, reaching people most affected by HIV with critical HIV prevention services, providing support for adherence to treatment and other essential health services, and advocating for resources and the human rights of people living with and affected by HIV.

Despite the wide recognition of the important role civil society plays in responding effectively to health and other related challenges, there continues to be decreased funding for communities. In addition, funding from international donors is too often channeled mainly through multilateral organizations. In most countries, this has negative implications for local civil societies and communities, hindering the full potential of community action and considerably reducing the amount of funding that reach the communities and their structures. According to the Global Humanitarian Assistance Report 2016, around two-thirds of funding from government donors was channeled through multilateral organizations in 2014 [Reference?]. Funding provided directly to local and national non-governmental organizations (NGOs) was only $0.4 \%$ of international humanitarian assistance in 2016. If the example of the Umunthu Foundation and the support from the AVERT is to be emulated, more challenges faced in the response to HIV and AIDS can be addressed.

Some study findings have revealed challenges affecting the ART including Option $\mathrm{B}+$. Treatment adherence and retention, stigma among the women who decide not to disclose their HIV + status and initiation on ART to their spouses, lack of adequate psychological and material support, lack of systems to track down the lost-to-follow (as some patients provide untraceable or incorrect information on their physical addresses of contact), and lack of space in public health facilities which have a high volume of ART patients are some of the major challenges that affect the Option B+. The civil society contribution to complement with the government efforts in successful implementation of the Option $\mathrm{B}+$ is crucial as the two need each other as strong partners in addressing HIV and AIDS pandemic in general and the Option $\mathrm{B}+$ in particular. The need for this partnership is evidence-based as it is derived from several study findings and direct testimonies from the government health officials. The collaboration between the two partners is very useful as the communities continue accessing the available quality services. However, funding the good work of civil society organizations is the hub for positive and effective 
contributions the civil society can make to the government-initiated programs such as the Option $\mathrm{B}+$ which the government of Malawi rolled out.

\section{Acknowledgements}

The UMUFO would like to express its heartfelt thanks and gratitude to AVERT of the United Kingdom for rolling out resources through grants to support the work the Foundation is doing in Bangwe and Limbe townships in Blantyre since 2010. Without their support, the organization could not have managed to implement this successful project in its supported areas.

In a special way, UMUFO would also like to thank the Ministry of Health (MoH) of the Government of Malawi through the Blantyre District Health Office and the HIV and AIDS Department for the cordial working partnership with UMUFO and technical support rendered to the organization which made it possible for implementing its successful project in its catchment areas.

\section{REFERENCES}

[1] Nations U. ten targets: 2011 United Nations political declaration on HIV and AIDS. Global progress and lessons learned, 2011 - 2015. UNAIDS; 2015.

[2] Lisa O'Brien; Nathan Shaffer et al. The incremental cost of switching from Option B to Option B + for the prevention of mother-to-child transmission of HIV. Bulletin of the World Health Organization. 2014; p. 162-170.

[3] Ciaranello AL; Perez F; Keatinge J. WHO 2010 guidelines for prevention of mother-to-child HIV transmission in Zimbabwe: modeling clinical outcomes in infants and mothers. PLosOne. 2011 .

[4] Schouten E. Management of Health Sciences Website. [Online].; 2012.. Available from:

https://www.msh.org/news-events/stories/option-b-in-malawi -the-origins-and-implementation-of-a-global-health-innovatio n.

[5] Leach lemens. AidsMap. [Online]. 2013 [cited 2917. Available from:

http://www.aidsmap.com/Malawi-achieves-seven-fold-increa se-in-ART-for-pregnant-and-breastfeeding-women/page/2596 $284 /$.
[6] Chimbwandira F, Mhango E, Makombe S, Midiani D et al. Impact of an innovative approach to prevent mother-to-child transmission of HIV-Malawi. Morbidity and Mortality Weekly Report. ; 2013.

[7] Tenthani L, Haas A, Tweya H, Van Oosterhout, Chimbwandira $\mathrm{F}$ et al. Retention in care under universal antiretroviral therapy for HIV infected pregnant and breastfeeding women (Option B+) in Malawi. AIDS. 2014; 28(4): p. 589-598.

[8] Schnack A, Rempis E, Decker S, Braun V, Rubaihayo J, Busingye $\mathrm{P}$ et al. Prevention of mother-to-child transmission of HIV in Option B+ era: Uptake and adherence during pregnancy in western Uganda. AIDS Patient Care STDs. 2016; 30(3): p. 110-118.

[9] Napúa M PJCFHRMJMCea. Option B+ in Mozambique: Formative Research Findings for the Design of a Facility-Level Clustered Randomized Controlled Trial to Improve ART Retention in Antenatal Care. J Acquir Immune Defic. 2016; 72: p. 181-188.

[10] David O. Umunthu Foundation Website. [Online].; 2015. Available from: http://umunthufoundationmw.com/.

[11] Salem G,Katy P, Hannock T, Sam Phiri et al. Exploring factors associated with ART adherence and retention in care under Option B+ strategy in Malawi: A qualitative study. PLoS One. 2017 June.

[12] Busza J, Walker D, Hairston A, Gable A, Pitter C, Lee S et al. Community-based approaches for prevention of mother to child transmission in resource-poor settings: a social ecological review. J Int AIDS Soc. 2012; 15(4).

[13] Dunlap J, Foderingham N, Bussell S, Wester C, Audet C, Aliyu M. Male Involvement for the Prevention of Mother-to-Child HIV Transmission: A Brief Review of Initiatives in East, West, and Central Africa. Curr HIV/AIDS Rep. 2014; 11[/2]: p. 109-118.

[14] Kalembo F, Zgambo M, Mulaga A, Yukai D, Ahmed N. Association between Male Partner Involvement and the Uptake of Prevention of Mother-to-Child Transmission of HIV (PMTCT) Interventions in Mwanza District, Malawi: A Retrospective Cohort Study. PLOS ONE. 2013; 8.

[15] L. SKaM. Barriers to initiating antiretroviral therapy during pregnancy: a qualitative study of women attending services in Cape Town, South Africa. 2012;11[/2]. Afr J AIDS Res. 2012; $11[/ 2]$ : p. $65-73$.

[16] Nachega J, Knowlton A, Deluca A, Schoeman J, Watkinson L, Efron A et al. Treatment Supporter to Improve Adherence to Antiretroviral Therapy in HIV-Infected South African Adults. J Acquir Immune Defic Syndr. 2006; 43(Supplement 1): p. 127-133. 